7-1994

\title{
Transfer Function Matrix Identification from Input-Output Frequency Response Data
}

Zhiqiang Gao

Cleveland State University, Z.GAO@csuohio.edu

BoH\&ew Trabachanił ditional works at: https://engagedscholarship.csuohio.edu/enece_facpub Cleveland State University

Part of the Electrical and Computer Engineering Commons

Razzañ $V_{\text {Sacescu }}$ to this work benefit you? Let us know!

Cleveland State University

Publisher's Statement

NOTICE: this is the author's version of a work that was accepted for publication in Journal of the Franklin Institute-Engineering and Applied Mathematics. Changes resulting from the publishing process, such as peer review, editing, corrections, structural formatting, and other quality control mechanisms may not be reflected in this document. Changes may have been made to this work since it was submitted for publication. A definitive version was subsequently published in Journal of the Franklin Institute-Engineering and Applied Mathematics, 331, 4, (07-01-1994); 10.1016/0016-0032(94)90007-8

\section{Original Citation}

Gao, Z., Tabachnik, B., , \& Savescu, R. (1994). Transfer function matrix identification from inputoutput frequency response data. Journal of the Franklin Institute, 331(4), 435-448. doi:10.1016/

0016-0032(94)90007-8

\section{Repository Citation}

Gao, Zhiqiang; Tabachnik, Bruce; and Savescu, Razvan V., "Transfer Function Matrix Identification from Input-Output Frequency Response Data" (1994). Electrical Engineering \& Computer Science Faculty Publications. 55. https://engagedscholarship.csuohio.edu/enece_facpub/55

This Article is brought to you for free and open access by the Electrical Engineering \& Computer Science Department at EngagedScholarship@CSU. It has been accepted for inclusion in Electrical Engineering \& Computer Science Faculty Publications by an authorized administrator of EngagedScholarship@CSU. For more information, please contact library.es@csuohio.edu. 


\title{
Transfer Function Matrix Identification from Input-Output Frequency Response Data
}

\author{
by ZHIQIANG GAO, BRUCE TABACHNIK and RAZVAN V. SAVESCU \\ Department of Electrical Engineering, Cleveland State University, Cleveland, \\ OH 44115, U.S.A.
}

\begin{abstract}
A new formulation of transfer function matrix identification in frequency domain is introduced. It reduces the problem to a simple linear least square problem. It is shown that such a system identification problem is a special case of a matrix interpolation problem and much insight can be obtained by examining its algebraic characteristics. A new approach is proposed to determine the transfer function matrix of a multi-input and multi-output system from the input-output data. It eliminates the common assumption in the literature that the frequency response of the system is given. Its efficiency and practicality is superior to the existing methods, where the solution is obtained by solving a nonlinear least square problem using mathematical programming techniques. The simplicity of the new procedure makes it a viable candidate for real time implementation where systems can be identified on-line. Unmodeled dynamics can also be better characterized.
\end{abstract}

\section{Introduction}

One of the classical problems in system identification is to determine transfer functions from the frequency response of linear time-invariant systems (Fig. 1). Typically, the data acquisition mechanism takes a time record of input and output data and transforms it to frequency domain, $y\left(j \omega_{i}\right)$ and $u\left(j \omega_{i}\right)$, where $i=1,2, \ldots$. For single-input and single-output (SISO) systems, the frequency response is obtained as the ratio of $y\left(j \omega_{i}\right)$ and $u\left(j \omega_{i}\right)$, i.e. $\tilde{G}\left(j \omega_{i}\right)=y\left(j \omega_{i}\right) / u\left(j \omega_{i}\right) i=1,2, \ldots$ Then, the system identification problem becomes that of finding a transfer function, $G(s)$, whose frequency response approximates $\widetilde{G}\left(j \omega_{i}\right) i=1,2, \ldots$, with minimal error. This is also known as the curve fitting problem where the measurements are fitted by a transfer function, $G(s)$, parameterized as the ratio of the denominator and numerator polynomials. The coefficients of the two polynomials are determined to minimize the cost function

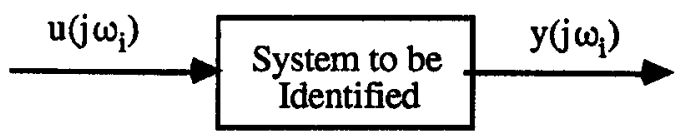

FIG. 1. System identification in frequency domain. 


$$
J=\sum_{i} w_{i}\left|G\left(j \omega_{i}\right)-\widetilde{G}\left(j \omega_{i}\right)\right|^{2}
$$

where $w_{i}$ represents the weights at different frequencies. Note that this is a nonlinear least square problem since the relation between the error, $G\left(j \omega_{i}\right)-\tilde{G}\left(j \omega_{i}\right)$, and the denominator coefficients of $G(s)$ is nonlinear.

Several curve fitting algorithms have been proposed in the literature for SISO identification (1-7). Various gradient decent optimization techniques were used to solve the nonlinear least square problem. The problem was first investigated as a least square problem in (1). A relatively simple and effective algorithm, known as the SK algorithm or SK iteration, was proposed in (2). Orthogonal polynomial basis was introduced to improve the numerical robustness $(4,8)$. Attempts were also made to extend the results for SISO systems to multi-input and multi-output (MIMO) systems (8-10). The curve fitting problem for MIMO systems was formulated; given the frequency response of the system, $\tilde{G}\left(j \omega_{i}\right) i=1,2, \ldots$, find the transfer function matrix $G(s)$ such that the cost function

$$
J=\sum_{i} w_{i}\left\|G\left(j \omega_{i}\right)-\tilde{G}\left(j \omega_{i}\right)\right\|_{f}^{2}
$$

is minimized (9). Here $\|\cdot\|_{f}$ represents the Frobenious norm, that is $\|X\|_{f}^{2}=$ $\operatorname{Tr}\left\{X^{*} X\right\}$ where "*" denotes complex conjugate transpose. An algorithm based on the SK iteration and Gauss and Newton algorithm (11) was proposed in (9) in which the problem was treated as a general optimization problem of the form

$$
\min _{\theta}\|f(\theta)\|_{2}^{2}
$$

where $f(\theta)$ is a nonlinear function of the parameter vector $\theta$. Another proposed approach (10) for MIMO system identification is to reduce it to a sequence of SISO system identifications. Each transfer function in the transfer function matrix is identified individually using the SISO system approach. The MIMO system is identified by minimal realization of the identified transfer functions.

The recent results on matrix interpolation (12) provide an effective mathematical tool to study the system identification problem. The rational matrix interpolation problem is defined to represent a $(p \times m)$ rational matrix $H(s)$ by interpolation triplets or points $\left(s_{i}, a_{i}, b_{i}\right) i=1, l$ which satisfy

$$
H\left(s_{i}\right) a_{i}=b_{i} \quad i=i, \ldots, l
$$

where $s_{i}$ are complex scalars and $a_{i} \neq 0, b_{i}$ complex $(m \times 1),(p \times 1)$ vectors respectively. The system identification problem studied here can be seen as a particular type of interpolation problem where the interpolation triplets, $\left(s_{i}, a_{i}, b_{i}\right) i=1, l$, are replaced by the measurements triplets, $\left(j \omega_{i}, u\left(j \omega_{i}\right), y\left(j \omega_{i}\right)\right) i=1, l$. The matrix interpolation theory provides a mathematical framework in which the algebraic aspects of the identification problem can be examined.

In this paper, a novel methodology is proposed for system identification in frequency domain. A new formulation of this classical system identification prob- 
lem is introduced, which allows various aspects of the problem to be examined by using matrix interpolation theory (12). More insight to the problem is shown. A new computer algorithm is developed to determine transfer function matrix for both SISO and MIMO systems. Two major benefits of the investigation are the vastly improved efficiency and the practicality of the new algorithm. In the new problem formulation, the coefficients of the transfer function matrix are linearly related to the error and they can be obtained by solving a linear least square problem. The new algorithm is more practical and therefore easier to implement, especially for MIMO systems. Instead of requiring the frequency response of the system to be identified, $\widetilde{G}\left(j \omega_{i}\right) i=1,2, \ldots$, it will only need the input and output data in frequency domain, that is $y\left(j \omega_{i}\right)$ and $u\left(j \omega_{i}\right)$, where $i=1,2, \ldots$ This is significant, especially for MIMO systems, since, unlike $\widetilde{G}\left(j \omega_{i}\right), y\left(j \omega_{i}\right)$ and $u\left(j \omega_{i}\right)$ are directly available from the measurements. Better characterization of unmodeled dynamics can also be obtained.

The main results are discussed in Section II, the implementation of the new approach and illustrative examples are given in Section III, and some concluding remarks are given in Section IV.

\section{Main Results}

The current results in literature on system identification from frequency response all require that the frequency response of the system to be identified, $\widetilde{G}\left(j \omega_{i}\right)$, where $i=1,2, \ldots$, is given. In practice, however, only the input and output are measurable. For SISO systems, this does not pose much difficulty as one can always take the ratio of $y\left(j \omega_{i}\right)$ and $u\left(j \omega_{i}\right)$ to obtain the frequency response $\widetilde{G}\left(j \omega_{i}\right)$. Unfortunately, it is not so trivial for MIMO systems considering all possible couplings between various inputs and outputs. Therefore, the assumption that $\tilde{G}\left(j \omega_{i}\right)$ is given seems very restrictive and impractical, particularly for MIMO systems.

The nature of the system identification problem dictates that one must work with the measurements $y\left(j \omega_{i}\right)$ and $u\left(j \omega_{i}\right)$, instead of $\widetilde{G}\left(j \omega_{i}\right)$. Ideally, the transfer function matrix $G(s)$ should be determined such that it fits the measurements as follows

$$
G\left(j \omega_{i}\right) u\left(j \omega_{i}\right)=y\left(j \omega_{i}\right), \quad \text { where } i=1,2, \ldots .
$$

Note that (5) applies to both SISO and MIMO systems. For SISO systems $G(j \omega)$, $u(j \omega)$ and $y(j \omega)$ are scalars; while for MIMO systems $G(j \omega)$ is a matrix; $u(j \omega)$ and $y(j \omega)$ are vectors. The problem of interest is to determine $G(s)$ such that the error, $y\left(j \omega_{i}\right)-G\left(j \omega_{i}\right) u\left(j \omega_{i}\right)$, is minimized in some sense. Note that the error defined here is more practical than the one used in (1) and (2) because it does not require the knowledge of the actual frequency response matrix, $\widetilde{G}(j \omega)$.

It is usually more convenient to deal with a polynomial matrix than a rational matrix. Assuming $G(s)$ is a $p \times m$ rational matrix, let $G(s)=D^{-1}(s) N(s)$ be a left coprime fraction representation (13), where $D(s)$ and $N(s)$ are $(p \times p)$ and $(p \times m)$ polynomial matrices, respectively. Clearly, equation (4) is equivalent to 


$$
N\left(j \omega_{i}\right) u\left(j \omega_{i}\right)=D\left(j \omega_{i}\right) y\left(j \omega_{i}\right), \quad \text { where } i=1,2, \ldots
$$

and the error can be defined as

$$
E_{i}=N\left(j \omega_{i}\right) u\left(j \omega_{i}\right)-D\left(j \omega_{i}\right) y\left(j \omega_{i}\right), \quad \text { where } i=1,2, \ldots
$$

Now the problem can be formulated as follows.

\subsection{Problem formulation}

Given column degrees of $N(s)$ and $D(s)$, and the input and output measurements, $u\left(j \omega_{i}\right)$ and $y\left(j \omega_{i}\right)$, where $i=1,2, \ldots$, find a proper transfer function matrix, $G(s)=D^{-1}(s) N(s)$, such that the cost function

$$
J=\|E W\|_{f}
$$

is minimized. Here, the matrix $W=\operatorname{diag}\left\{w_{1}, w_{2}, \ldots\right\}$, is a diagonal weighting matrix where $w_{i}$ reflects the weight at frequency $\omega_{i} ; E$ is the error matrix defined as

$$
E=\left[E_{1}, E_{2}, \ldots\right] .
$$

Note that the column degrees of $N(s)$ must not be greater than those of $D(s)$ for a proper solution transfer function matrix to exist.

The new formulation is fundamentally different from the existing ones described in equations (1) and (2). A critical characteristic of (8) is that the relationship between the coefficients in $N(s)$ and $D(s)$ and the cost function is linear. This is shown in the following.

First, let equation (6) be rewritten as

$$
\left[N\left(j \omega_{i}\right),-D\left(j \omega_{i}\right)\right]\left[\begin{array}{l}
u\left(j \omega_{i}\right) \\
y\left(j \omega_{i}\right)
\end{array}\right]=\underline{0}, \quad \text { where } i=1,2, \ldots .
$$

Given the column degrees of $[N(s),-D(s)], d_{i} i=1, p+m$, the polynomial matrix $[N(S)-D(S)]$ can be represented as

$$
[N(s),-D(s)]=[N,-D] S(s)
$$

where $S(s)$ represents the matrix polynomial basis in a block diagonal form and $[N,-D]$ contains all the corresponding coefficients

$$
S(s)=\left[\begin{array}{cccc}
1 & & & \\
s & & & \\
\cdots & & & \\
s^{d_{1}} & & & \\
& 1 & & \\
& s & & \\
& \cdots & & \\
& s^{d_{2}} & & \\
& & \cdots & \\
& & & s \\
& & & \cdots \\
& & & s^{d_{p+m}}
\end{array}\right] .
$$

Assuming there are $l$ measurements $\left\{j \omega_{i}, u\left(j \omega_{i}\right), y\left(j \omega_{i}\right)\right\}$, let $S_{l}$ be 


$$
S_{l}:=\left[S\left(j \omega_{1}\right)\left[\begin{array}{l}
u\left(j \omega_{1}\right) \\
y\left(j \omega_{1}\right)
\end{array}\right], \ldots, S\left(j \omega_{l}\right)\left[\begin{array}{l}
u\left(j \omega_{l}\right) \\
y\left(j \omega_{l}\right)
\end{array}\right]\right]
$$

all individual equations in (10) can be put in a single matrix equation as

$$
[N,-D] S_{l}=\underline{0} .
$$

The error associated with each frequency $\omega_{i}$, defined as $E_{i}$ in Eq. (7), can now be written as

$$
E_{i}=[N,-D] S\left(j \omega_{i}\right)\left[\begin{array}{l}
u\left(j \omega_{i}\right) \\
y\left(j \omega_{i}\right)
\end{array}\right]
$$

It is obvious that

$$
E=[N,-D] S_{l}
$$

Thus, it is shown that the coefficients of the rational matrix to be identified are linearly related to the error matrix $E$.

From Eq. (15), the problem simply reduces to a linear least square problem: find $[N,-D]$ such that $J=\|E W\|_{f}$ is minimized. This applies to both SISO and MIMO systems. Furthermore, only the input and output data, $u\left(j \omega_{i}\right)$ and $y\left(j \omega_{i}\right)$, where $i=1,2, \ldots$, are needed to determine the transfer function matrix.

To better understand the problem and to develop a new algorithm, a number of issues must be addressed. For example, under what conditions does the system identification problem described in (5) and (6) have exact solutions? Is the problem in the new formulation numerically ill-conditioned? Is there a way to improve the numerical robustness? How many measurements should be taken to identify a transfer function matrix of certain order? For MIMO systems, can one take more than one measurement at the same frequency with different combination of inputs and outputs? etc. These issues have not been studied in depth mainly because of the lack of an appropriate mathematical framework. The recent development in matrix interpolation theory provides such a framework.

\subsection{Additional constraints}

For MIMO systems, the appropriate solutions must satisfy the conditions that $D(s)$ is nonsingular and $G(s)=D^{-1}(s) N(s)$ is proper. This can be achieved by imposing additional linear constraints in the form of

$$
[N,-D] P=Q
$$

where $P$ and $Q$ are constant matrices with appropriate dimensions. For example, since $D(s)$ is a $p \times p$ matrix, $p$ additional constraints can be used to make the leading coefficient matrix of $D(s)$ a nonsingular matrix, say, a $p \times p$ identity matrix, $I_{p}$. In this case the equation to be solved becomes

$$
[N,-D]\left[S_{l}, P\right]=\left[0, I_{p}\right] .
$$

Furthermore, this will also guarantee the properness of the transfer function matrix 
since the column degrees of $D(s)$ in the solution can now be selected to be greater than or equal to those of $N(s)$.

\subsection{Existence of exact solutions and minimum number of measurements required}

It is shown above that the system identification problem can be formulated as a polynomial matrix interpolation problem. That is, given the measurements data $\left\{\omega_{i}, u\left(j \omega_{i}\right), y\left(j \omega_{i}\right), i=1,2, \ldots\right\}$, find the polynomial matrix $[N(s),-D(s)]$ which satisfies Eq. (10) with $D(s)$ nonsingular. The coefficients of $[N(s),-D(s)]$ can be determined numerically from (17). The solution of (17) is unique if $\left[S_{l}, P\right]$ has full rank. Therefore, we have the following theorem.

\section{Theorem 1}

Given $\left\{\omega_{i}, u\left(j \omega_{i}\right), y\left(j \omega_{i}\right), i=1, l\right\}$ and nonnegative integers $d_{i}=\operatorname{deg}_{c i}[N(s)$, $-D(s)]$ with $l=\left(\Sigma d_{i}\right)+m$, and the $P$ matrix where $[N,-D] P=I_{p}$, such that the $\left(\Sigma d_{i}+m+p\right) \times(l+p)$ matrix $\left[S_{l}, P\right]$ has full rank, there exists a unique $(p \times(m+p))$ polynomial matrix $[N(s),-D(s)]$ with ith column degree equal to $d_{i}$, where $i=1$, $m$ and leading coefficients of $D(s)$ an identity matrix, for which Eq. (10) is satisfied.

Note that the unique solution $[N(s),-D(s)]$ leads to a unique $p \times m$ transfer function matrix, $G(s)=D^{-1}(s) N(s)$, with $I_{p}$ as the leading coefficients matrix of $D(s)$. For such a rational matrix to be uniquely identified, $l=\Sigma d_{i}+m$ number of measurements are required. It is of interest to examine what happens when $l$ is different from the required number. That is what happens when $l \neq \Sigma d_{i}+m$ :

The equation of interest is $[N,-D]\left[S_{l}, P\right]=\left[0, I_{p}\right]$ in (17). A solution $[N,-D]$ $\left(p \times\left(\Sigma d_{i}+m+p\right)\right)$ of this equation exists if and only if

$$
\operatorname{rank}\left[\begin{array}{cc}
S_{l}, & P \\
0, & I_{p}
\end{array}\right]=\operatorname{rank}\left[S_{l}, P\right] .
$$

This implies that there exists a unique solution $[N,-D]$ if and only if rank $\left[S_{l}, P\right]=l+p$, that is if and only if $\left[S_{l}, P\right]$, a $\left(\Sigma d_{i}+m+p\right) \times(l+p)$ matrix, has full column rank.

(i) When $l>\Sigma d_{i}+m$, the system of equations in (17) is over specified; there are more equations than unknowns. If the additional $\left(l-\left(\Sigma d_{i}+m\right)\right)$ equations are linearly dependent upon the previous $\left(\sum d_{i}+m\right)$ ones, then a $[N(s),-D(s)]$ with column degrees $d_{i}$, where $i=1, m+p$ is uniquely determined provided that $\left(\Sigma d_{i}+m\right)$ measurements $\left\{\omega_{i}, u\left(j \omega_{i}\right), y\left(j \omega_{i}\right)\right.$ satisfy the conditions of the Theorem. Otherwise an exact solution does not exist.

(ii) When $l<\Sigma d_{i}+m,[N(s),-D(s)]$ with column degrees $d_{i}$, where $i=1, m+p$ is not uniquely specified, since there are more unknowns than Eqs in (17). That is, in this case there are many matrices $[N(s),-D(s)]$ with the same column degrees $d_{i}$ which satisfy the $l$ interpolation constraints.

\subsection{Choice of measurements}

For SISO systems, it is known that the measurements $\left\{j \omega_{i}, u\left(j \omega_{i}\right), y\left(j \omega_{i}\right)\right\}$ should be taken at distinct frequencies to avoid redundancy. This is not necessarily true 
for MIMO systems. Consider the $S_{l}$ matrix in (13), a measurement is redundant if the corresponding column in $S_{l}$ is linearly dependent on the previous columns. It is shown (12) that in general $j \omega_{i}$, where $i=1, l$ do not have to be distinct; repeated values for $j \omega_{i}$, coupled with different $u\left(j \omega_{i}\right)$ will still produce full rank in $S_{l}$ in many instances. It was also shown that $S_{l}$ has full rank for almost any $u\left(j \omega_{i}\right)$ when $j \omega_{i}$ are distinct.

This is significant in system identification because it provides an alternative to frequency weighting. In the classical approaches, the frequencies of importance were given larger weight so that the errors at these frequencies will be smaller. This is shown in Eqs (1) and (2). In the new approach, besides frequency weighting, one can also use more than one measurement at a particular frequency. Therefore, the transfer function matrix can be made more accurate for a set of input patterns at the frequency of interests.

\subsection{Numerical issues}

It is well known that the standard polynomial basis $\left\{1, s, s^{2}, \ldots\right\}$ sometimes lead to poor numerical conditions in system identification especially when the frequency range of the data is rather wide and the order of the plant is relatively high. This problem can be dealt with by using various orthogonal polynomials, such as Chebychev polynomials, as basis. The change of basis can be carried out with ease in the framework of (11)-(14). Let $[N(s),-D(s)]$ be expressed as

$$
[N(s),-D(s)]=[N,-D]_{T} T(s)
$$

where $[N,-D]_{T}$ is the representation of $[N(s),-D(s)]$ with respect to orthogonal polynomials $\left\{t_{0}(s), t_{1}(s), t_{2}(s), \ldots\right\}$ and

$$
T(s)=\left[\begin{array}{cccc}
t_{0}(s) & & & \\
t_{1}(s) & & & \\
\ldots & & & \\
t_{d_{1}}(s) & & & \\
& t_{0}(s) & & \\
& t_{1}(s) & & \\
& \ldots & & \\
& t_{d_{2}}(s) & & \\
& & \ldots & \\
& & & t_{0}(s) \\
& & & t_{1}(s) \\
& & & t_{d_{p+m}}(s)
\end{array}\right] .
$$

Let $T_{l}$ be defined similarly as $S_{l}$

$$
T_{l}:=\left[T\left(j \omega_{1}\right)\left[\begin{array}{l}
u\left(j \omega_{1}\right) \\
y\left(j \omega_{1}\right)
\end{array}\right], \ldots, T\left(j \omega_{l}\right)\left[\begin{array}{l}
u\left(j \omega_{l}\right) \\
y\left(j \omega_{l}\right)
\end{array}\right]\right]
$$

then, $[N,-D]$ can be found by solving 


$$
[N,-D]_{T} T_{l}=\underline{0} .
$$

Once $[N,-D]_{T}$ is found, $[N(s),-D(s)]$ can be determined from (18).

\subsection{Measurement noise}

In practice, whenever a measurement is taken, the presence of noises is inevitable. The noises may be white noise or colored noise depending on the plant and the way measurements are taken. For a system of low pass nature, the response to high frequency input is more susceptible to noises than to the low frequency input. As a result, the data in low frequency range is more reliable than that in high frequency range. Frequency weighting can be used here to reflect the confidence in each measurement. It could also be used to reflect the importance of the accuracy of the model at each frequency. Various othogonal polynomial basis that lead to better numerical properties will also help to reduce the sensitivity to the presence of noise.

\subsection{Unknown system order}

In system identification, the order of the model may not be readily available. In fact order estimation is a problem of its own. This is especially challenging in MIMO system identification. In the framework proposed above, an intuitive approach can be used to deal with this difficulty. First, from the frequency response data, the lower bound of the system order can be estimated. This bound can be used as a starting point in the search for the transfer function matrix of the lowest degree that yields reasonably small error. The system identification algorithm can be executed repeatedly with the increasing column degrees of $[N(s),-D(s)]$. It should only stop when it reaches a point where the error is smaller than a predetermined value, or, the increase in the column degrees does not bring significant decrease in the error.

\subsection{Discrete-time system identification in frequency domain}

Discrete-time systems have transfer functions as rational functions of $Z$-transform variable $z$. The frequency response of discrete-time systems is obtained by substituting $z$ as $z=\mathrm{e}^{j \omega T}$, where $T$ is the sampling period. The problem of system identification in frequency domain for discrete-time systems is similarly defined: given the measurement triplets $\left\{j \omega_{i}, u\left(\mathrm{e}^{j \omega_{i} T}\right), y\left(\mathrm{e}^{j \omega_{i} T}\right)\right\}$, find a transfer function matrix $G(z)$ which satisfies

$$
G\left(\mathrm{e}^{j \omega_{i} T}\right) u\left(\mathrm{e}^{j \omega_{i} T}\right)=y\left(\mathrm{e}^{j \omega_{i} T}\right), \quad \text { where } i=1,2, \ldots
$$

Based on the approach described above for the continuous-time systems, a new algorithm for discrete-time system identification will be developed. Note that unlike the polynomial basis for continuous-time system, $\left\{1, j \omega,(j \omega)^{2}, \ldots\right\}$, the basis for discrete-time system is $\left\{1, \mathrm{e}^{j \omega T},\left(\mathrm{e}^{j \omega T}\right)^{2}, \ldots\right\}$, where all elements in the basis have the magnitude of one. Consequently, it seems that the discrete-time formulation has better numerical properties. This is especially true for systems with large bandwidth. 


\subsection{Real-time implementation}

Many applications, such as self-tuning and fault-tolerant control systems, require that the mathematical model of the plant be identified in real-time. The proposed approach will lead to a numerically efficient computer algorithm which is quite suitable for such purposes. In the problem formulation introduced above, the system identification problem is reduced to solving a set of linear equations in least square sense. Therefore, the solution can be obtained by solving these linear equations simultaneously. The existing results can not fully meet the requirements due to their overwhelming computational complexity.

\subsection{Characterization of uncertainty}

Recent work in systems and control theory has bred methodologies which result in controllers with guaranteed robustness and performance for a given mathematical model of a physical system. For these guarantees to hold on the actual system, the mathematical model must describe the behavior of the actual physical systems to be controlled including variations, perturbations, and external noises. A model that meets such criteria is referred to as a robust model (14). The difference between the actual system, $\tilde{G}(s)$, and the model obtained from system identification, $G(s)$, is known as the uncertainty, or unmodeled dynamics, denoted as $\Delta(s)=\tilde{G}(s)-G(s)$. It is important that the uncertainty be characterized so that the information can be taken into account in the design process. In general, the control system can be made more robust if we know more details about the unmodeled dynamics. Due to limitations in the existing approach, the uncertainty can only be represented by upper and lower bound on $\|\Delta(j \omega)\|$, where $\|\cdot\|$ represents matrix norms. With the new framework for system identification introduced above, a novel approach to fully characterize the uncertainty will be developed.

Since $\widetilde{G}(s)=G(s)+\Delta(s)$ is the actual transfer function matrix of the system to be identified, it satisfies the measurements exactly

$$
\left(G\left(j \omega_{i}\right)+\Delta\left(j \omega_{i}\right)\right) u\left(j \omega_{i}\right)=y\left(j \omega_{i}\right), \quad \text { where } i=1, l .
$$

With $G(s)$ obtained, it is desirable to characterized $\Delta(s)$. Equation (23) can be rewritten as

$$
\Delta\left(j \omega_{i}\right) u\left(j \omega_{i}\right)=\hat{y}\left(j \omega_{i}\right), \quad \text { where } i=1, l
$$

where $\hat{y}\left(j \omega_{i}\right)=y\left(j \omega_{i}\right)-G\left(j \omega_{i}\right) u\left(j \omega_{i}\right)$. One way to characterize the uncertainty is to find $\Delta(s)$ such that it satisfies (24). Note that, according to the matrix interpolation theory (12), one can almost always choose the order of $\Delta(s)$ high enough so that it solves all Eqs in (24) exactly.

One may wonder if $\Delta(s)$ can be determined exactly, why should it not be included in as part of the model, $G(s)$. The answer is two fold. First, since the behavior of a system may vary from one experiment to another, different $\Delta(s)$ may be obtained from different experiments. Thus, a set of $\Delta(s)$ can be used to represent the variations in the system. Secondly, the complexity of the model is another important feature to be considered. An identified model should be relatively simple and should minimally cover the experimental data set. The $\Delta(s)$ that satisfies all 
equations in (24) is likely to have a much higher order than that of the model. Therefore, including $\Delta(s)$ in the model will make it unnecessarily complex.

Once $\Delta(s)$, or a set of $\Delta(s)$, is obtained, the bound on the uncertainty can be determined by taking and plotting the matrix norm. Obviously $\Delta(s)$ contains much more information than its norm and the availability of such information will undoubtedly lead to the development of less conservative methodology in robust control design.

\section{Implementation and Examples}

Matlab programs have been developed based on the new approach discussed above. Some implementation issues are discussed below. Numerical examples are also given.

\subsection{An alternative basis}

Although the formulation introduced above allows one to specify the column degrees of each column in $[N(s)-D(s)]$, it is usually not necessary for system identification purposes. To simplify the procedure in the implementation, an alternative basis is used and is discussed below.

Assume that the highest degrees of any term in $N(s)$ and $D(s)$ are $d_{n}$ and $d_{d}$, respectively. Then, $N(s)$ and $D(s)$ can be written in a matrix polynomial form:

$$
N(s)=N_{0} s^{0}+N_{1} s^{1}+\cdots+N_{d_{n}} s^{d_{n}}=N\left[s^{0} s^{1} \ldots s^{d_{n}}\right]^{\prime}
$$

and

$$
D(s)=D_{0} s^{0}+D_{1} s^{1}+\cdots+D_{d_{n}} s^{d_{n}}=D\left[s^{0} s^{1} \ldots s^{d_{d}}\right]^{\prime}
$$

where $N=\left[N_{1} N_{2} \ldots N_{d_{n}}\right], D=\left[D_{1} D_{2} \ldots D_{d_{d}}\right]$. Thus, the polynomial matrix $N(s),-D(s)]$ can be represented as

$$
[N(s),-D(s)]=[N-D] S(s)
$$

where

$$
S(s)=\left[\begin{array}{cc}
I_{m} s^{0} & \\
I_{m} s & \\
\cdots & \\
I_{m} s^{d_{n}} & \\
& I_{p} s^{0} \\
& I_{p} s \\
& \cdots \\
& I_{p} s^{d_{d}}
\end{array}\right]
$$

$S_{l}$ is similarly obtained as

$$
S_{l}:=\left[S\left(j \omega_{1}\right)\left[\begin{array}{l}
u\left(j \omega_{1}\right) \\
y\left(j \omega_{1}\right)
\end{array}\right], \ldots, S\left(j \omega_{l}\right)\left[\begin{array}{l}
u\left(j \omega_{l}\right) \\
y\left(j \omega_{l}\right)
\end{array}\right]\right]
$$

The solution can be found by solving 
TABLE I

Measurement data for Example 1

\begin{tabular}{lcccccccccc}
\hline$\omega(\mathrm{Hz})$ & 1 & 3 & 7 & 10 & 15 & 20 & 25 & 30 & 35 & 40 \\
\hline$|G(j \omega)|$ & 1 & 0.95 & 0.77 & 0.70 & 0.67 & 0.63 & 0.60 & 0.53 & 0.48 & 0.44 \\
$\arg (G(j \omega))$ & -2 & -13 & -24 & -35 & -44 & -57 & -62 & -71 & -75 & -87 \\
\hline$\omega(\mathrm{Hz})$ & 50 & 60 & 70 & 80 & 90 & 100 & 110 & 120 & 130 & 140 \\
$|G(j \omega)|$ & 0.35 & 0.31 & 0.33 & 0.35 & 0.32 & 0.32 & 0.30 & 0.29 & 0.27 & 0.26 \\
$\arg (G(j \omega))$ & -87 & -110 & 92 & -105 & -119 & -128 & -145 & -156 & -166 & -172 \\
\hline
\end{tabular}

$$
[N,-D] S_{l}=\underline{0} .
$$

In addition, to guarantee that $D(s)$ is nonsingular, the leading coefficient matrix of $D(s), D_{d_{d}}$, is forced to be an $p \times p$ identity matrix, $I_{p}$. Equation (30) is now equivalent to

$$
\left[N,-D_{1}\right] S_{l 1}=B_{l}
$$

where $D_{1}$ can be found as $D_{1}=\left[D_{1} D_{2} \ldots D_{d_{d-1}}\right]$ and $S_{l 1}$ and $B_{l}$ are derived from the partition of $S_{l}, S_{l}=\left[\begin{array}{c}S_{l 1} \\ B_{l}\end{array}\right]$.

To obtain the coefficient matrix $\left[N,-D_{1}\right]$ from (31), a least square solution is sought. Note that since $S_{l}$ is a complex matrix, the solution may also be a complex matrix. A real solution can be determined from

$$
\left[N,-D_{1}\right]\left[\operatorname{Re}\left(S_{l 1}\right), \operatorname{Im}\left(S_{l 1}\right)\right]=\left[\operatorname{Re}\left(B_{l}\right), \operatorname{Im}\left(B_{l}\right)\right] .
$$

Based on the above discussion, Matlab programs have been developed to carry out the system identification, and full details can be found in $(\mathbf{1 5}, \mathbf{1 6})$.

Example 1. Experimental frequency response data in Table I was used to test the Matlab program for SISO system identification. The data was collected from experiments on a supersonic jet engine (7). The transfer function obtained by the our Matlab algorithm is

$$
G(s)=\frac{-16.34 s^{2}+1374.88 s+193461.16}{s^{3}+122.89 s^{2}+15424.51 s+211949.42} .
$$

The frequency response generated from this transfer function compared to the measurement data can be seen in Fig. 2 . A very close fit is clearly shown. Note that this third order transfer function is significantly simpler than the best result obtained in (7), which shows a similar frequency response but has an order of 5.

\subsection{Evaluation of the MIMO system identification algorithm}

Due to the lack of experimental data in the literature, the computer program is tested on the input-output data generated from a given transfer function matrix, 

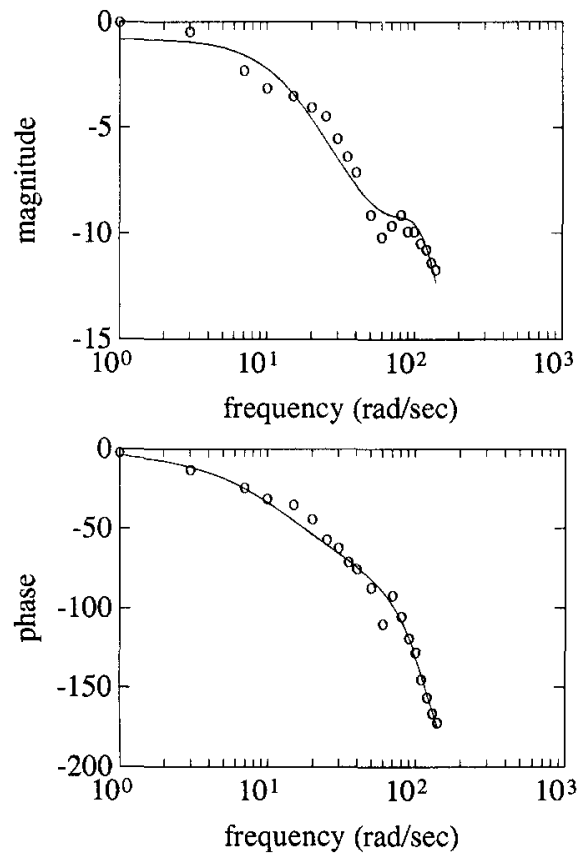

FIG. 2. Frequency response from the data and the transfer function model in Example 1: - frequency response of the identified transfer function; measurement data.

$\tilde{G}(s) . u\left(j \omega_{i}\right)$ is generated randomly with uniform distribution. $y\left(j \omega_{i}\right)$ is calculated as $y\left(j \omega_{i}\right)=\tilde{G}\left(j \omega_{i}\right) u\left(j \omega_{i}\right)$. The new approach proposed here for a MIMO system identification is rather unique in that it does not require the knowledge of the actual frequency response of the system, i.e. $\widetilde{G}\left(j \omega_{i}\right) i=1,2, \ldots$ Therefore, the frequency response generated from the transfer function matrix, $G\left(j \omega_{i}\right) i=1$, $2, \ldots$, obtained by the algorithm can not be directly compared to $\tilde{G}\left(j \omega_{i}\right)$. To evaluate the accuracy of the new algorithm, the output response generated from the transfer matrix identified, $\hat{y}\left(j \omega_{i}\right)=G\left(j \omega_{i}\right) u\left(j \omega_{i}\right)$, is compared to the actual data, $y\left(j \omega_{i}\right)$.

Example 2. A $2 \times 2$ transfer function matrix is given as

$$
\widetilde{G}(s)=\left[\begin{array}{cc}
s+1 & 0 \\
1 & s+2
\end{array}\right]^{-1}\left[\begin{array}{ll}
s & 2 \\
0 & 1
\end{array}\right]=\left[\begin{array}{cc}
\frac{s}{s+1} & \frac{2}{s+1} \\
\frac{-s}{(s+1)(s+2)} & \frac{s-1}{(s+1)(s+2)}
\end{array}\right] .
$$

From matrix interpolation theory, $l=\Sigma d_{i}+m+p=7$ measurements can be used to uniquely determine this transfer function matrix. Seven frequencies $\omega_{i} i=1,7$ are arbitrarily chosen within the range of zero to one radian/second. Seven arbitrary inputs, $u\left(j \omega_{i}\right) i=1,7$, are arbitrarily generated and the output response is calculated 
TABLE II

Measurement data for Example 2

\begin{tabular}{lcccc}
\hline$\omega(\mathrm{rad})$ & 0.1000 & 0.1429 & 0.1857 & 0.2286 \\
$u_{1}(j \omega)$ & $13.3881-16.4769 j$ & $-15.9598+4.9172 j$ & $-7.5992-1.4061 j$ & $-4.3337-2.6746 j$ \\
$u_{2}(j \omega)$ & $12.2230-20.1499 j$ & $-10.6773-15.5498 j$ & $4.2099+2.4494 j$ & $7.0625-5.7025 j$ \\
$y_{1}(j \omega)$ & $21.9778-41.1587 j$ & $-26.2890-29.6239 j$ & $9.0176+1.8129 j$ & $11.3121-14.9811 j$ \\
$y_{2}(j \omega)$ & $-4.3413+10.7215 j$ & $8.2663+6.446 j$ & $-2.3540+.5369 j$ & $-1.5740+4.8192 j$ \\
\hline
\end{tabular}

\begin{tabular}{lccc}
\hline$\omega(\mathrm{rad})$ & 0.2714 & 0.3143 & 0.3571 \\
$u_{1}(j \omega)$ & $2.2786-1.8727 j$ & $1.3986-6.3885 j$ & $-6.2897-6.2448 j$ \\
$u_{2}(j \omega)$ & $-10.1699+12.0856 j$ & $-7.4809+6.0554 j$ & $13.9483+5.7223 j$ \\
$y_{1}(j \omega)$ & $-12.2038+28.1021 j$ & $-8.1996+15.1274 j$ & $29.6323-1.3848 j$ \\
$y_{2}(j \omega)$ & $-0.0686-7.9989 j$ & $-0.3449-4.4818 j$ & $-6.9847+4.8008 j$
\end{tabular}
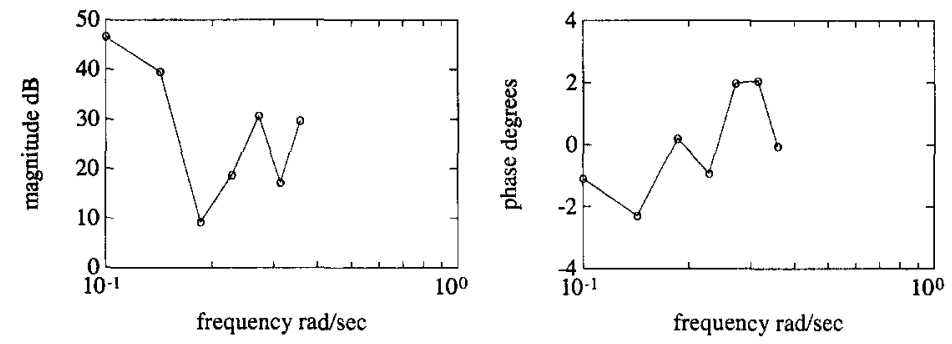

(a) $\hat{y}_{1}\left(j \omega_{i}\right)$ and $y_{1}\left(j \omega_{i}\right)$
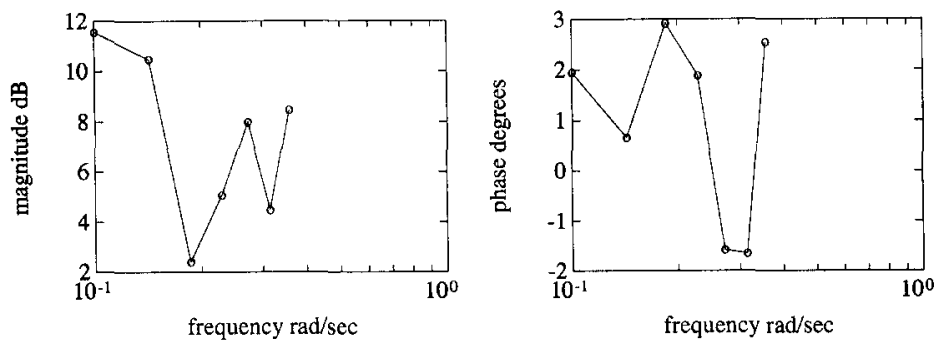

(b) $\hat{y}_{2}\left(j \omega_{i}\right)$ and $y_{2}\left(j \omega_{i}\right)$

$$
\begin{aligned}
& \text {-: frequency response of the identified transfer function } \\
& 0 \text { : measurement data }
\end{aligned}
$$

FIG. 3. Output frequency response $\hat{y}\left(j \omega_{i}\right)$ and $y\left(j \omega_{i}\right):-$ frequency response of the identified transfer function; measurement data.

using Eq. (5). The data is listed in Table II. From this set of data, the computer program determined $G(s)$ with the error between the coefficients of $\widetilde{G}(s)$ and $G(s)$ in the range of $10^{-15}$. The closeness of $\hat{y}\left(j \omega_{i}\right)$ and $y\left(j \omega_{i}\right)$ can be seen in Fig. 3. 


\section{Conclusion}

A new mathematical framework is introduced for MIMO system identification in frequency domain. The new approach is vastly improved on the efficiency and practicality and suitable for on-line implementation. The algorithm determines the transfer function matrix from the input-output data instead of the frequency response data of the system; thus it eliminates the conventional assumption that the frequency response of the system is given. A computer program is developed to determine the transfer function matrix of multi-input and multi-output systems by solving a linear least square problem.

\section{References}

(1) E. C. Levy, "Complex curve fitting", IEEE Trans. Aut. Control, Vol. AC-4, pp. $37-$ 43, 1959.

(2) C. K. Sanathanan and J. Koerner, "Transfer function synthesis as a ratio of two complex polynomials", IEEE Trans. Aut. Control, Vol. 8, pp. 56-58, 1991.

(3) R. C. Seidel, "Transfer function parameter estimation from frequency response dataA Fortran program", NASA Tech. Memo. Washington, D.C., X-3286, Sept. 1975.

(4) J. L. Adcock, "Curve fitter for pole-zero analysis", Hewlett-Packard Journal, Jan. 1987.

(5) M. D. Sidman, F. E. DeAngelis and G. C. Verghese, "Parametric system identification on logarithmic frequency response data", IEEE Trans. Aut. Control, Vol. 36, No. 9, pp. 1065-1070, 1991.

(6) D. M. Tilly, "Three algorithms for parametric frequency domain system identification", Lawrence Livermore National Lab. Rep. UCID-20255, Nov. 1984.

(7) J. T. Spanos, "Algorithms for $l_{2}$ and $l_{\infty}$ transfer function curve fitting", AIAA Guidance, Navigation and Control Conference, New Orleans, LA, August, 1991.

(8) R. L. Dailey, and M. S. Lukich, "MIMO transfer function curve fitting using Chebyshev polynomials", SIAM 35th Anniversary Meeting, Denver, CO, 1987.

(9) D. S. Bayard, "Multivariable frequency domain identification via 2-norm minimization", Proceedings of the American Control Conference, 1992.

(10) P. L. Lin and Y. C. Wu, "Identification of multi-input multi-output linear systems from frequency response data", Trans. ASME, J. Dynamic Systems, Measurement, and Control, Vol. 104, pp. 58-64, 1982.

(11) P. Gill, W. Murray and M. H. Wright, "Practical Optimization", Academic Press, New York, 1981.

(12) P. Antsaklis and Z. Gao, "Polynomial and rational matrix interpolation: theory and control applications", Int. J. Control, Vol. 58, No. 2, pp. 349-404, 1993.

(13) C. T. Chen, "Linear System Theory and Design", Holt, Rinehart and Winston, Fort Worth, 1984.

(14) W. Wang and A. N. Moser, "Modeling of undertain systems-problems and solutions", Proceedings of 1992 American Control Conference, Chicago, pp. 291-295. 1992.

(15) Bruce Tabachnik, "A computer algorithm for multi-input multi-output system identification using Chebychev polynomials", M.S. thesis, Department of Electrical Engineering, Cleveland State University, May 1994.

(16) R. V. Savescu, "System identification from frequency response using matrix interpolation theory", M.S. thesis, Department of Electrical Engineering, Cleveland State University, October 1992. 